\title{
Assessment of the Sanitary Conditions of Catering Establishments and Food Safety Knowledge and Practices of Food Handlers in Addis Ababa University Students' Cafeteria
}

\author{
Asrat Meleko ${ }^{1}$, Andualem Henok ${ }^{1,}$, , Worku Tefera ${ }^{2}$, Tafesse Lamaro ${ }^{3}$ \\ ${ }^{1}$ Department of Public Health, Mizan-Tepi University, Mizan, Ethiopia \\ ${ }^{2}$ School of Public Health Addis Ababa University, Addis Ababa, Ethiopia \\ ${ }^{3}$ Department of Nursing, Mizan-Tepi University, Mizan, Ethiopia \\ Email address: \\ andualemhenok@gmail.com (A. Henok)
}

\section{To cite this article:}

Asrat Meleko, Andualem Henok, Worku Tefera, Tafesse Lamaro. Assessment of the Sanitary Conditions of Catering Establishments and Food Safety Knowledge and Practices of Food Handlers in Addis Ababa University Students' Cafeteria. Science Journal of Public Health. Vol. 3, No. 5, 2015, pp. 733-743. doi: 10.11648/j.sjph.20150305.30

\begin{abstract}
Background: Every institution which provides food for a large group of consumers has the responsibility to keep the safety and wholesomeness of food otherwise it may result outbreaks of food borne illness. The most identified contributing factors of food borne illness in mass catering establishments were cross contamination, dirty work environment and poor personal hygiene practice by food handlers. Objective: The objective of this study was to assess the sanitary conditions of catering establishments and food safety knowledge and practices of food handlers in Addis Ababa University students' cafeterias. Methods: A cross-sectional study was conducted from January, 2014 to May, 2014. A total of 12 student catering establishments from 7 campuses were studied for sanitary condition of premises and a total of 302 food handlers were assessed for knowledge and practice statuses. For data collection questionnaires and appropriate checklists were used. Bacteriological examination was made from all catering premises following appropriate standard procedures. The data was entered by using EPI INFO version 3.5.1 and cleaned before transferring to SPSS version 17 statistical packages which was used for data management and analysis. Results: Majority, 212(70.2\%), of food handlers were females and their median age were 29. Among the 302 subjects, 197 (65.2\%) of them had food hygiene training. All, 302 (100\%), of food handlers were literate and $283(93.7 \%)$ of them had adequate knowledge of food borne diseases. Twelve of premises had a clean wall and ceiling in their kitchen and dining rooms. Similarly all premises had openable window, adequate light and adequate ventilation. E. coli were not identified at all and in 1 (2.8\%) of swabbed utensils S. aureus were identified. Practice scores revealed that $52.3 \%$ of food handlers had a poor food handling practice. Conclusion: This study revealed that the repair and sanitary conditions of premises were in a good condition. Nearly half of food handlers had a poor food handling practice. Provision of training and basic sanitary facilities by management is recommended.
\end{abstract}

Keywords: Sanitary Condition, Food Handlers, Food Safety, Food Handling Practices

\section{Introduction}

Even though an adequate supply of safe and wholesome food is essential to the health and wellbeing of humans, there are plenty of conditions that food affects the health of people across the globe due to contamination. Even if the problem is worse in developing nations it is also common in developed countries. Even modern technological advance could not stop the occurrence of food related diseases $(1,2)$.
According to several studies a typical microbiological contamination of food occur in food and drink establishments due to dirty food contact surfaces, poor personal hygiene practices and inappropriate storage temperature (2).

Eating establishments in hospitals, universities, schools and restaurants provide meal service for a large number of consumers from the same source. Food prepared in large amount is vulnerable for contamination and may lead to the occurrence of food borne outbreaks unless basic sanitary 
practices are well maintained. Therefore, food establishment serving a large number of individuals are responsible to provide safe and wholesome food for their consumers (3).

As a result of eating unsafe food thousands of millions of people become ill and may die across the globe (4). Although the global incidence of food borne disease is difficult to estimate due to a number of factors, a report from $\mathrm{WHO}$ indicates that in 2008 alone an estimated of 1.8 million people died from diarrheal diseases across the globe in which a great proportion of these cases can be attributed to contamination of food and drinking water (4-6). Another study done in the United States indicates that, about 76 millions of illnesses, 325,000 hospitalizations, and 5,000 deaths are caused by foodborne pathogens each year (5). In developing countries, up to $70 \%$ of cases of diarrheal diseases are associated with the consumption of contaminated foods (6).

In absence of well-maintained and proper food handling practices mass catering establishments have the potential to impart disastrous effect on human health because of their scale and complexity. In 1969/70 there were about 737 outbreaks of food borne disease with 52,011 cases reported to the US centers for disease control and prevention. Of those $33 \%$ of outbreaks occurred in mass catering services (7).

In developing countries like Ethiopia, the problem attains great proportions due to many reasons, basic among which are poverty and lack of public health awareness. Relevant and well organized data regarding the magnitude of food borne disease in Addis Ababa University does not exist. However, according to the existing reports from students' clinic there were repeated occurrence of intestinal parasitic infections from students which could be attributed to consumption of unsafe food and water.

It is very crucial to ensure food safety in institutional catering services where comparatively large number of clients served on a regular basis since a single contamination may lead to outbreaks. The major institutional foodservice operations include hospital and long-term care, schools and college and university foodservices.

Based on current fact Ethiopia showed a great transformation regarding education, infrastructure, and other economic activity. Accordingly radical expansion of universities observed throughout the whole country. Therefore, it is very essential to ensure safe food handling and preparation practices because of protecting enrolled huge number of students in those universities from food borne diseases. However, in most universities data on sanitary condition of student catering establishments were not available.

Since there is no researches done in Addis Ababa University before, this study expected to draw attention to identifications of sanitary gaps in students cafeteria which will help the university administration to give emphasis and to allocate resources to improve the overall repair and sanitary conditions of premises and empowering and equipping food handlers with safe food handling practice working with in students' cafeteria.
Assessment of the sanitary conditions of food service establishments and food safety knowledge and practices of food handlers in Addis Ababa University

\section{Methodology}

\subsection{Study Area and Period}

The study area was Addis Ababa University, students' cafeteria. The University was found in Addis Ababa city, which is the capital city of Ethiopia. It has seven campuses; six in Addis Ababa and one in Bishoftu about 45 kilometers away. In 2009/10 there were 20,701 enrolled undergraduate students, 7,127 graduate students, and 14,669 continuing education students, making a total student body of 42,497 . The study was focused in six campuses which were found in Addis Ababa city. Accordingly around 302 food handlers are currently working in 6 different campuses which had a direct contact with food and food contact surfaces. The study was conducted from January, 2014 to February, 2014.

\subsection{Study Design}

The study design was cross-sectional study.

\subsection{Source Population}

All student catering establishments of Addis Ababa University were the source population.

\subsection{Study Population}

All students cafeterias of Addis Ababa University was the study subjects and all food handlers in all student catering services were enlisted for knowledge and practice assessment.

\subsection{Sample Size Determination}

There were a total of 302 food handlers in 12 food establishments. All 302 food handlers working in Addis Ababa University students' cafeteria were enrolled for knowledge and practice assessment.

For overall assessment of sanitary conditions of premises all 12 student cafeterias in seven campuses were included in the study. Microbiological swab test was done in all 12 student catering services of Addis Ababa University. Number of swabs taken was restricted to fit within the time, financial and resource parameters of the project. As a result of this three swabs were submitted from each student catering establishments. One swab was obtained from bowls and the remaining two swabs were obtained from plates. Therefore, a total of 24 swabs from plates and a total of 12 swabs from bowls were taken.

\subsection{Sampling Procedure}

First information was gathered about the number of cafeteria employees working in each campus to obtain sampling frame. Since the study includes all food handlers data was gathered from all employees according to the list. A 
total of 302 food handlers were selected from all campuses which had a direct contact with food related activity for knowledge and practice assessment.

Food utensils for bacteriological swab test examination selected from a group of utensils which were cleaned and sanitized and stored for direct use. All students' cafeterias were selected for bacteriological examination of food utensils.

\subsection{Data Collection Procedures}

Structured questionnaire and checklist were developed for the purpose of data collection after reviewing relevant literature and views of professionals in this area. The questionnaire was structured and designed to accommodate the response of respondents and the physical observation of data collectors and was designed to obtain information on sanitary condition of premises and knowledge and practice of food handlers. It was prepared originally in English and then translated into Amharic and back to English by two different Environmental health professionals in order to obtain content validity. Finally the questionnaire was administered in Amharic. Check list was prepared and used as a tool during swab sample collection. Standard swab sample collection and transportation procedure was implemented for microbiological food utensil examination. Environmental health and medical laboratory professionals were recruited for data collection and microbiological analysis of food utensils respectively. Data collectors and supervisors were trained and after the training the questionnaire was pretested in cafeterias that were not included in the actual study to ensure the quality and validity of data. Regular supervision and reviewing the completed questionnaire was carried out by two environmental health professionals and by the principal investigator daily to maintain data quality. For the qualitative method the data collectors used the pre designed checklist to probe the desired answers from the cafeteria managers.

The main purpose of bacteriological examination was assessing the effectiveness of cleaning and disinfecting food utensils and washing facilities. Collection of samples was followed a standard procedure and practice. Fifteen plate/bowls were selected from each student catering establishments for swab test. Samples were selected before lunch time since utensils were cleaned and sanitized as well they become ready for use for serving students during that time. Plates/bowls counted as they are in chairs and systematically the required amounts of sample units were recruited. Swabbing one sterilized cotton swab on wooden applicator stick was used for each group of sampling units (i.e. five plate/bowls) which were two groups of utensils in each of 12 student catering establishments. The assessment of effectiveness of cleaning and sanitizing food utensils was done based on the number of bacteria found per $\mathrm{cm}^{2}$. To cover an equivalent area of selected sampling units approximately $50 \mathrm{~cm}^{2}(2.5 \mathrm{~cm}$ by $20 \mathrm{~cm}, 5 \mathrm{~cm}$ by $10 \mathrm{~cm}$ or other dimensions) of them Roll or drag moist cotton swab across sample area slowly and firmly 3 times reversing the direction each time. Since samples must reach the laboratory in a microbiologically unchanged condition from the existing condition at the time of sampling contamination of the sample and microbial growth or death during sampling, transport and storage should be avoided. Therefore the samples were kept refrigerated in an ice box and were transported to the laboratory by considering the standard time which is between 24-48 hours in which the result would be valid. Then in the laboratory the swab samples were directly cultured into solid media of MacConkey, blood agar (BA) and yeast extract agar to isolate the possible organisms. The Petri dishes were incubated at $37^{\circ} \mathrm{C}$ for 48 hours to detect the presence of total coliform and similarly swab sample was also inoculated into test tubes containing MacConkey broth and incubated for $24-48$ hours at $37^{\circ} \mathrm{C}$ and $44^{\circ} \mathrm{C}$ to determine the growth of E.coli. Biochemical tests were performed according to the essence in the identification of bacteria species for further confirmation $(33,34)$

\subsection{Operational Definitions $(5,24,25)$}

Food and drink establishments: - is an institution which provides food and drink services to a relatively large number of users in the form of breakfast, lunch, dinner or beverages.

Food handler - any person who directly handles packaged or unpackaged food, food equipment and utensils, or food contact surfaces and is therefore expected to comply with food hygiene requirements

Food safety - assurance that food will not cause harm to the consumer when it is prepared and/or eaten according to its intended use

Food hygiene - all conditions and measures necessary to ensure the safety and suitability of food at all stages of the food chain.

Contaminant - any biological or chemical agent, foreign matter, or other substances not intentionally added to food which may compromise food safety or suitability.

Contamination - means to make unfit for use by the introduction or potential introduction of unwholesome or undesirable elements

Disinfection - the reduction, by means of chemical agents and/or physical methods, of the number of micro-organisms in the environment, to a level that does not compromise food safety or suitability.

Easily Cleanable - means surfaces which are readily accessible and fabricated of such materials and finishes that residue can be effectively removed by normal cleaning methods.

Food-Contact Surfaces - means those surfaces of equipment and utensils with which food normally comes in contact

Perishable food: a food that can easily spoil by microbial action e.g. meat, egg, etc

Non-perishable food: a food that can persist for a long period of time without spoilage e.g. flour, sugar etc

Personal hygiene - refers to those protection measures primarily with the responsibility of the individual, which promote and limit the spread of infectious disease, like hand 
washing using soap and water, keep body clean etc.

Good repair condition: - shall mean absence of big cracks or detached areas, holes and lack of painting for food preparation areas/kitchens, dining room or service room; and being free of breaks (open seams), corrosion, and cracks and easily cleanable for food utensils and equipments.

Adequate lighting: - is to mean that a healthy person (without major visual problem) can see or easily identify objects in the room comfortably without straining of the eye.

Adequate ventilation: - is to mean that a room is free of reasonably disagreeable odor and have at least one openable window.

Properly managed toilet: - shall mean when a toilet/latrine was found free of litters, tissue/anal cleansing paper, fly access and other dirty materials like faeces or urine around the latrine.

Proper storage :- When garbage/refuse are stored in receptacle which is durable, have tight fitting cover, moisture proof and light to lift and transport.

Cleanliness/clean: - shall mean absence of dust particles, grease, finger and other marks for food utensil and being free of spider webs, dust and smoke particles for kitchen and dining /service room.

Sign of spoilage: - means the change of the physical characteristics (color change, bad odor) of perishable foodstuffs that can be easily detected by observation.

Personal hygiene - refers to those protection measures primarily with the responsibility of the individual, which promote and limit the spread of infectious disease, like hand washing using soap and water, keep body clean etc.

Sanitary condition: means effective systems which help to ensure adequate and appropriate maintenance and cleaning, control pests, manage wastes and monitor effectiveness of maintenance and sanitation procedures

\subsection{Study Variables}

\subsubsection{Dependent Variable}

Sanitary conditions and availability of Sanitary facilities like,water supply, toilet facility,utensil cleanliness, hand washing basin availability, waste disposal mechanism Knowledge of food handlers

Hygienic practice of food handler Microbiological quality of food utensils

\subsubsection{Independent Variables}

Educational level, age and gender, work experience and training status of food handlers, income status of food handlers, average working hour per day, inspection status of establishments by experts

\subsection{Data Analysis}

The data was entered to EPI INFO version 3.5.1 and cleaned before transferring to SPSS version 17 statistical package which was used for data management and analysis. Cross tabulations and chi-squared tests $(5 \%$ significance level) were used to determine the influence of gender, age, educational level, and training status of food handlers on their practice score. Where cells had a value with less than 5, Fisher's exact test had to be used for testing significance. The score of practice was categorized as: good or poor based on the summation of individual scores of the variables. The score range was between $0-10$ and the scores were converted to 100 points and food handlers who had a score of above $70 \%$ were categorized as good and below it as poor. The variables used for scoring overall practice were Food handlers wear outer garments/gown during inspection, hair covered with appropriate aprons, wear finger ornaments during food handling, finger nail cut short, wash hands before starting food preparation today (this morning), cook foods thoroughly before ready for consumptions, check ingredients expiry date before using for food preparations, cover mouth with tissue paper when coughing or sneezing accidentally during food preparation, dry washed utensils before ready for use and use separate utensils for raw and cooked foods. The score range for knowledge of food handlers was between 0-4 and the scores were converted to 100 points and food handlers who had a score of below $75 \%$ were categorized as good and below it as poor. The variables used to score the knowledge of food handlers were knowledge about food borne disease, about cause of food borne disease, about mode of transmission for food borne disease and about cause of food contamination. Potential influencing factors towards practice were examined by using univariate and multivariate analyses. Variables that were found statistically significant in univariate analysis and those under main interests of the study were included in multivariate analysis. The results were presented by appropriate tabulations based on the determined variables, crude or adjusted odds ratio with $95 \%$ confidence interval and its corresponding $p$-values.

\subsection{Data Quality Management}

The principal investigators performed data entry and cleaning. The quality of data was ensured through training of data collectors, close supervision and prompt feedback, reviewing each of completed questionnaires daily and reinterviewing certain student's cafeterias randomly. Data consistency and completeness was made all the way during data collection, data entry and analysis. Two week earlier to the actual data collection period, pretest was conducted on 20 food handlers working in one of student's cafeteria of Addis Ababa University. Data collection facilitators and supervisors were trained for a day before and after pretest. Well experienced lab technician was recruited for microbiological analysis.

\subsection{Ethical Consideration}

The ethical approval and clearance were obtained from Research and Ethics committee of the school of public health, Addis Ababa University; formal letter of support was submitted to Addis Ababa University student's directorate office and respective cafeteria managers and permission was obtained. Data were collected with the consent of study participants after they were informed 
about the objective of the study, how long it takes the interview and the measurements. The identity of the respondent was protected by code and participants were reassured of the confidentiality of the information they provided. Advice regarding basics of personal hygiene and environmental sanitation were provided.

\section{Results}

\subsection{General Information About Managers and Study Units}

Based on the information obtained from Addis Ababa University student's directorate office there were a total of 12 student caterings in 7 different campuses. All those 12 student caterings were included under the study subjects. The average number of students consumed in each catering establishment was 2011 with the minimum number of 1184 and maximum of 2700 students. In the past six months 10 of student catering establishments were inspected by sanitarian and according to the manager response inspections done in all 10 student caterings were with education and it was beneficial for the establishment. About 8 of managers were male and the remaining 4 were females. Their median age was 39 with the minimum and maximum age of 30 and 42 respectively. All managers completed a higher education program.

Table 1. Socio demographic conditions of managers and general information of student caterings of Addis Ababa University, 2014 ( $n=12)$.

\begin{tabular}{ll|l}
\hline Variable & Frequency & Percent (\%) \\
\hline Age (years) & & \\
$30-40$ & 10 & 83.3 \\
$>40$ & 2 & 16.7 \\
Sex & & \\
Male & 8 & 66.7 \\
Female & 4 & 33.3 \\
Educational status & & \\
12 & 12 & 100.0 \\
Cafeteria inspected past 6 month & & \\
Inspected & 10 & 83.3 \\
Not inspected & 2 & 16.7 \\
Inspection with education and beneficial & 10 & 100.0 \\
Number of students consumed per day & & \\
1001-2000 & 4 & 33.3 \\
Above 2000 & 8 & 66.7 \\
\hline
\end{tabular}

\subsection{Socio-Demographic Profile of Food Handlers}

According to the information obtained from the employee's roster there were a total of 302 food handlers working in all 12 student caterings. Fortunately, all food handlers were on duty during data collection time and therefore the non response rate was zero. Majority of food handlers, 212 (70.2), were females and 90(29.8) were males. Nearly half, 156 (51.7\%), of them were aged between 22 and 29 and their median age were 29 with a minimum and maximum age of 22 and 47 respectively. About 156(45\%) of them were educated from grade 9-10 and 153 (50.7\%) of them were married. Almost all, 296(98\%), of food handlers had an income ranged between 501 and 1000 birr and their mean work experience were about 2.41 years. (Table 2)
Table 2. Socio-demographic profile of food handlers in student caterings of Addis Ababa University, 2014, $(n=302)$.

\begin{tabular}{lll}
\hline Variable & Frequency & Percent (\%) \\
Age (years) & 156 & \\
$19-29$ & 138 & 41.7 \\
$30-40$ & 8 & 2.6 \\
$>40$ & & \\
Sex & 90 & 29.8 \\
Male & 212 & 70.2 \\
Female & & \\
Educational status & 1 & 0.3 \\
Grade 1-6 & 77 & 25.5 \\
Grade 7-8 & 136 & 45 \\
Grade 9-10 & 88 & 29.1 \\
Grade 11-12 & & \\
Income status (Birr) & 6 & 2.0 \\
Below 500 & 296 & 98.0 \\
501-1000 & & \\
Marital status & 128 & 42.7 \\
Single & 153 & 50.7 \\
Married & 12 & 4.0 \\
Divorced & 5 & 1.7 \\
Widowed & 4 & 1.3 \\
Separated & & 60.6 \\
Work experience (years) & 183 & 31.8 \\
Below 2 year & 96 & \\
2-4 year & 23 & \\
Above 2 year & & \\
\hline & & \\
\hline
\end{tabular}

\subsection{Physical and Repair Conditions of Kitchen and Dining Rooms}

\subsubsection{Kitchen}

All, 12, student catering establishments inspected based on a pre designed checklist. Accordingly, 11 of the premises had kitchen floor without any cracks and detached areas. About 11 of premises' kitchens' wall and ceiling were free of any dust, smoke or other dirt and 11of them have a wall and ceiling without any cracks and detached areas. All 12 had openable window, adequate light and adequate ventilation during inspection. Similarly all kitchens of student caterings are free of infestation with any vectors and rodents.

\subsubsection{Dining Room}

Table 3. Sanitary and physical conditions of dining room and kitchens of student caterings of Addis Ababa University, $2014(n=12)$.

\begin{tabular}{ll}
\hline Variable & Frequency \\
\hline Kitchen (n=12) & 11 \\
Floor had no cracks and detached areas & 11 \\
Wall and ceiling clean & 11 \\
Wall and ceiling had no cracks and detached areas & 11 \\
Had openable windows & 12 \\
Had adequate light & 12 \\
Had adequate ventilation & 12 \\
Had no any infestation of vectors and rodents & \\
Dining room (n=12) & 12 \\
Floor had no cracks and detached areas & 11 \\
Wall and ceiling clean & 11 \\
Wall and ceiling had no cracks and detached areas & 12 \\
Had openable windows & 12 \\
Had adequate light & 12 \\
Had adequate ventilation & 11 \\
Had no any infestation of vectors and rodents & \\
\hline
\end{tabular}


All, 12, student catering dining rooms were in a good repair condition. Majority, 11 , of them had a clean wall and ceiling. About 11 of premises dining room had a wall and ceiling without any cracks and detached areas. All, 12, of dining rooms have openable windows, adequate light and adequate ventilation. Most 11 of dining rooms were not infested with any vectors and rodents during inspection time.

\subsection{Food Storage and Refrigerator Condition}

About 11 of premises had storage room for perishable and nonperishable foods and from those establishments 9 of them have free space and sufficient air circulation in the room. All, 12 , of establishments had a walking type of refrigerator. All 12 of those premises had not a refrigerator with a fixed thermometer. In most, 9, of the premises there was no any sign of spoilage on stored foods.

\subsection{Sanitary Facilities and Water Supply}

\subsubsection{Sanitary Facilities}

\section{(i). Latrine and Lavatory Services}

All, 12 of establishments had toilet facility and nearly all, 11 of them use dry pit latrine type. Majority, 8 of those latrine facilities were clean and of them were open for use during inspection time. All, 12, of establishments desludge by vacuum truck when latrine is full. About 8 of student catering establishments lack urinals. Lavatory facilities were available in 7 of establishments and in all of them it was separated for food handlers and other customers. In 6 of those establishments with lavatory service there were detergent during inspection and almost all 7 of them had a fixed wash basin type. All, 7 of establishments with lavatory service were open for service during inspection. Nearly all, 11 of catering establishments use drainage system for disposal of waste water form kitchen and hand washing services.

\section{(ii). Solid Waste Management}

Half, 6 of the premises had container for temporary storage facility and all of them use barrel type. All, 6, of those establishments had a durable, tight fitting and light to carry solid waste storage container. About 5 of them placed the solid waste storage container at appropriate site during inspection. In all, 12, of student catering establishments the final disposal of solid waste undertaken using municipal tractor/lorry.

\subsubsection{Accessibility of Water and Dish Washing Facilities}

Nearly all, 11, of inspected student catering establishments had adequate running water in kitchen and all, 12, of them had both cold and running water at the same time. Majority, 10 , of establishments use machine to wash soiled dish and 2 of them wash manually. Those 2 of establishments who wash utensils manually was compartmentalized with 3 sinks. Nearly all, 11, of student caterings used hot water and detergents for washing dishes

\subsection{Knowledge and Practice of Food Handlers}

\subsubsection{Knowledge of Food Handlers About Food Borne Disease, Its Cause and Route of Transmission}

About 283(93.7\%) of food handlers were aware of at least one type of food borne disease. Main source of information were sanitarian during inspection and mass media, 136(45\%) and $128(42.4 \%)$ of them respectively. Nearly half, $202(66.9 \%)$, of food handlers had responded germs as cause of disease and majority $245(81.1 \%)$ of them knew that contaminated food could be the main route of transmission for food borne disease. About 214 (70.9\%) and 152 (50.3\%) of food handlers aware of that dirty hand and dirty utensils are the main means of food contamination respectively. According to overall score, the knowledge of food handlers regarding food borne disease, its cause, mode of transmission and cause of food contamination were good (Table-4).

Table 4. Knowledge of food handlers regarding food borne disease, mode of transmission and way of food contamination in student caterings of Addis Ababa University, 2014.

\begin{tabular}{lll}
\hline Variable & Frequency & Percent (\%) \\
\hline Knowledge of food handlers about food borne disease & \\
Yes & 283 & 93.7 \\
No & 19 & 6.3 \\
Source of information about FBD* & & \\
Health center & 100 & 33.1 \\
Sanitarian during inspection & 136 & 45.0 \\
Mass media & 128 & 42.4 \\
School & 82 & 27.2 \\
Response on cause of FBD* & & \\
Germs & 202 & 66.9 \\
Chemicals & 74 & 24.5 \\
Do not know & 50 & 16.6 \\
Knowledge about route of transmission for FBD* & \\
Contaminated food & 245 & 81.1 \\
Contaminated water & 91 & 30.1 \\
Vectors & 89 & 29.5 \\
Do not know & 18 & 6.0 \\
Knowledge on cause of food contamination* & & \\
Dirty hand & 214 & 70.9 \\
Infected food handler & 91 & 30.1 \\
Dirty utensils & 152 & 50.3 \\
Vectors & 83 & 27.5 \\
Dirty work environment & 104 & 34.4 \\
Do not know & 18 & 6.0 \\
\hline
\end{tabular}

NB. Those with asterisk (*) will not add up to $100 \%$ because of multiple responses

\subsubsection{Practice of Food Handlers}

About 210(69.5\%) of food handlers worn gown during inspection among those who had gown 136(45\%) of them kept their gown clean. Half, 160(53\%), of them covered hair with restraints and $179(59.5 \%)$ of them did not worn any finger ornaments during inspection time. About 219 (72.5\%) of food handlers' finger nail trimmed properly and almost all, $295(97.7 \%)$, of them wash hands always before starting any activity. About $152(50.3 \%)$ of food handlers check ingredients expiry date before they use them. Nearly half, 137 (45.4\%), of food handlers had not done medical checkup in the past one year and 141(44.4\%) of them did checkup 
before one year ago. Most, 197(65.2\%), of them took training on basics food safety before they were recruited in this establishment (Table 5).

Table 5. Food handling practice of food handlers in student caterings of Addis Ababa University, 2014

\begin{tabular}{|c|c|c|}
\hline Variable & Frequency & Percent (\%) \\
\hline \multicolumn{3}{|c|}{ Food handlers worn white gown during inspection } \\
\hline Yes & 210 & 69.5 \\
\hline No & 92 & 30.5 \\
\hline \multicolumn{3}{|c|}{ Food handler's gown } \\
\hline Clean & 136 & 45.0 \\
\hline Not clean & 74 & 24.5 \\
\hline \multicolumn{3}{|c|}{ Food handler's hair covered } \\
\hline Yes & 160 & 53.0 \\
\hline No & 142 & 47.0 \\
\hline \multicolumn{3}{|c|}{ Food handlers wear finger ornaments during food } \\
\hline \multicolumn{3}{|c|}{ Preparation } \\
\hline Yes & 123 & 40.7 \\
\hline No & 179 & 59.3 \\
\hline \multicolumn{3}{|c|}{ Food handlers finger nail cut short } \\
\hline Yes & 219 & 72.5 \\
\hline No & 83 & 27.5 \\
\hline \multicolumn{3}{|c|}{ Food handlers wash hands frequently before any event } \\
\hline Yes & 295 & 97.7 \\
\hline No & 7 & 2.3 \\
\hline \multicolumn{3}{|c|}{ Food handlers check expiry date of ingredients } \\
\hline Yes & 152 & 50.3 \\
\hline No & 150 & 49.7 \\
\hline \multicolumn{3}{|c|}{$\begin{array}{l}\text { Food handlers take training regarding food preparation and } \\
\text { Handling }\end{array}$} \\
\hline Yes & 197 & 65.2 \\
\hline No & 105 & 34.8 \\
\hline
\end{tabular}

According to the result displayed in table 6 there was statistically significant difference in the number of trained and untrained food handlers with regard to practices of wearing gown, washing hands before any food handling event, checking expiry date before consumptions, drying washed utensils before ready for use and using separate utensils for raw and cooked food.

Table 6. Food handler's hygienic practice in relation to training status of student catering services of Addis Ababa University, 2014.

\begin{tabular}{|c|c|c|c|c|}
\hline Practice of food handlers & Training & status & & \\
\hline & \multicolumn{2}{|c|}{ of food handlers } & & \\
\hline & Yes & No & \multicolumn{2}{|c|}{ OR $(95 \% \mathrm{CI})$} \\
\hline \multicolumn{5}{|l|}{ Food handlers wear gown } \\
\hline Yes & 142 & 68 & $\begin{array}{l}1.46 \\
9\end{array}$ & $\begin{array}{l}(1.886 \\
2.437)\end{array}$ \\
\hline No & 54 & 38 & 1.00 & \\
\hline \multicolumn{5}{|l|}{ Food handlers hair covered } \\
\hline Yes & 103 & 57 & $\begin{array}{l}0.95 \\
2\end{array}$ & $\begin{array}{l}(0.593 \\
1.529)\end{array}$ \\
\hline No & 93 & 49 & 1.00 & \\
\hline \multicolumn{5}{|c|}{ Food handlers wear finger ornaments during food preparations } \\
\hline Yes & 71 & 52 & $\begin{array}{l}0.59 \\
0\end{array}$ & $\begin{array}{l}(0.365 \\
0.953)\end{array}$ \\
\hline No & 125 & 54 & 1.00 & \\
\hline \multicolumn{5}{|c|}{ Food handlers cut finger nails short } \\
\hline Yes & 140 & 79 & $\begin{array}{l}0.85 \\
4\end{array}$ & $\begin{array}{l}(0.500 \\
1.460)\end{array}$ \\
\hline No & 56 & 27 & 1.00 & \\
\hline \multicolumn{5}{|c|}{ Food handlers wash hands before food handling event } \\
\hline Yes & 194 & 101 & 4.80 & $(2.915$ \\
\hline
\end{tabular}

\begin{tabular}{|c|c|c|c|c|}
\hline Practice of food handlers & Training & status & & \\
\hline & \multicolumn{2}{|c|}{ of food handlers } & & \\
\hline & Yes & No & \multicolumn{2}{|c|}{ OR $(95 \% \mathrm{CI})$} \\
\hline & & & 2 & $25.189)$ \\
\hline No & 2 & 5 & 1.00 & \\
\hline \multicolumn{5}{|c|}{ Cook foods thoroughly before Consumptions } \\
\hline Yes & 187 & 96 & $\begin{array}{l}2.16 \\
4\end{array}$ & $\begin{array}{l}(0.851 \\
5.505)\end{array}$ \\
\hline No & 9 & 10 & 1.00 & \\
\hline \multicolumn{5}{|c|}{ Check expiry date before food preparations } \\
\hline Yes & 110 & 42 & $\begin{array}{l}1.94 \\
9\end{array}$ & $\begin{array}{l}(1.205, \\
3.152)\end{array}$ \\
\hline No & 86 & 6 & 1.00 & \\
\hline \multicolumn{5}{|c|}{ Dry washed utensils before ready for use } \\
\hline Yes & 178 & 86 & $\begin{array}{l}2.30 \\
0\end{array}$ & $\begin{array}{l}(1.157, \\
4.571)\end{array}$ \\
\hline No & 18 & 20 & 1.00 & \\
\hline \multicolumn{5}{|c|}{ Use separate utensils for raw and cooked Food } \\
\hline Yes & 191 & 87 & $\begin{array}{l}8.34 \\
3\end{array}$ & $\begin{array}{l}(3.017 \\
23.071)\end{array}$ \\
\hline No & 5 & 19 & 1.00 & \\
\hline
\end{tabular}

Based on the summation of individual scores $52.3 \%$ of food handlers had a poor food handling practice. Individual who scored 7 out of 10 practice questions were categorized under good food handling practice. (Table-7)

Table 7. Food handling practice score of food handlers in Addis Ababa University, 2014.

\begin{tabular}{lll}
\hline Practice score & Frequency & Percent (\%) \\
\hline Good & 144 & 47.7 \\
Poor & 158 & 52.3 \\
Total & 302 & 100.0 \\
\hline
\end{tabular}

On Bivariate analysis, there was significance difference between good and poor practice regarding educational status, work experience, sex and training status of food handlers. Age of food handlers did not influenced the practice score of food handlers (Table 8).

Table 8. Univariate analysis between socio demographic profile of food handlers and food hygiene practice score, Addis Ababa University, 2014.

\begin{tabular}{llll}
\hline $\begin{array}{l}\text { Socio-demographic } \\
\text { variables }\end{array}$ & & Overall practice score & P-value \\
\hline & Good & Poor & 0.236 \\
\hline Age (years) & 68 & 88 & \\
$19-29$ & 73 & 65 & \\
$30-40$ & 3 & 5 & 0.030 \\
$>40$ & & & \\
Educational status & 0 & 1 & \\
Grade 1-6 & 32 & 45 & 0.037 \\
Grade 7-8 & 62 & 74 & \\
Grade 9-10 & 50 & 38 & 0.016 \\
Grade 11-12 & & & \\
Work experience & 80 & 103 & 0.005 \\
Below 2 year & 64 & 55 & \\
Above 2 year & & & \\
Sex & 38 & 52 & \\
Male & 106 & 106 & \\
Female & & & \\
Training status & 105 & 91 & \\
Trained & 39 & 67 & \\
Not trained & & & \\
\hline
\end{tabular}

On multivariate analysis variables that were found as 
significant potential influencing factors were sex of food handlers with $\mathrm{AOR}=1.66,95 \% \mathrm{CI}=1.395-2.123, \mathrm{P}=$ 0.012 , training status of food handlers with $\mathrm{AOR}=1.752$, $95 \% \mathrm{CI}=1.056,2.907, \mathrm{P}=0.03$ and educational status predominantly mainly secondary school (between grade 9 and 10) with $\mathrm{AOR}=1.497,95 \% \mathrm{CI} 1.250-2.987, \mathrm{P}=0.046$ (Table 9).

Table 9. Multivariate analysis between significant variables and food handling practice, Addis Ababa University, 2014.

\begin{tabular}{ll|l}
\hline Socio-demographic variables & Adjusted OR (95\% CI) & P-value \\
\hline Sex & & \\
Male & 1.0 & - \\
$\begin{array}{l}\text { Female } \\
\text { Work experience }\end{array}$ & $1.666(1.395,2.123)$ & 0.012 \\
$\begin{array}{l}\text { Below 2 year } \\
\text { Above 2 year }\end{array}$ & 1.0 & - \\
$\begin{array}{l}\text { Training status } \\
\text { Trained }\end{array}$ & $0.742(0.436,1.264)$ & 0.273 \\
Not trained & $1.752(1.056,2.907)$ & 0.030 \\
Educational status & 1.0 & - \\
Grade 1-6 & & \\
Grade 7-8 & 0.00 & 0.263 \\
Grade 9-10 & 0.00 & 1.00 \\
Grade 11-12 & $1.497(1.250,2.987)$ & 0.046 \\
Age (years) & $0.750(0.424,1.326)$ & 0.322 \\
$\begin{array}{l}\text { 19-29 } \\
\text { 30-40 }\end{array}$ & 0.00 & 0.335 \\
Above 40 & $1.125(0.240,5.280)$ & 0.882 \\
\hline
\end{tabular}

\subsection{Bacteriological Examination of Food Utensils}

From all 12 student catering services which are found in 7 campuses a total of 36 sample swabs were collected 3 sample swabs (each sample swab comprise of 5 food utensils) from each establishment. These swab samples consisted of 28 $(77.8 \%)$ swabs from the eating plates and $8(22.2 \%)$ swabs from the drinking bowls. ISO procedures for microbiological analysis and data collection of food utensils were used in the laboratory.

Cleaned food utensils stored on table with a cover in all, $12(100 \%)$, of student catering establishments. The repair condition and cleanliness of the food utensils as observed by naked eye, $29(80.6 \%)$ were new and tidy and the remaining $7(19.4 \%)$ were old, stained and cracked.

Table 10. Bacteriological swab test of food utensils in all student catering establishments of Addis Ababa University, $2014 \mathrm{~N}=36$.

\begin{tabular}{ll|l}
\hline Type of identified microorganisms & Frequency & Percent (\%) \\
\hline Aerobic mesophilic bacteria, colony/plate & & \\
No growth & 26 & 72.2 \\
$1-25$ & 5 & 13.9 \\
$26-50$ & 3 & 8.3 \\
$51-100$ & 1 & 2.8 \\
$>100$ & 1 & 2.8 \\
Fecal coliforms, MPN/100ml & & \\
No growth & 29 & 80.6 \\
$1-10$ & 3 & 8.3 \\
$11-50$ & 2 & 5.5 \\
$51-250$ & 1 & 2.8 \\
$500-1100$ & 1 & 2.8 \\
\hline
\end{tabular}

\begin{tabular}{lll}
\hline Type of identified microorganisms & Frequency & Percent (\%) \\
\hline Eschericia coli & 0 & 0 \\
Staphylococcus aureus & 1 & 2.8 \\
\hline
\end{tabular}

Aerobic mesophilic bacteria, E. coli and S. aureus as well as total and faecal coliforms were isolated. Aerobic mesophilic bacteria were grown in $10(27.8 \%)$ of the swabbed utensils with an average of 37 colonies per utensils, but only $1(2.8 \%)$ of the swabbed utensils were found above the acceptable level of aerobic mesophilic bacteria $(>100$ colonies/utensils). S. aureus were isolated from 1(2.8\%) of swabbed utensils and E. coli were not detected from all the swabbed utensils. Acid and gas formation in 7(19.4\%) of the examined utensils would also confirm the presence of total and faecal coliform.

\section{Discussion}

All parts of the premises including the floor, wall and ceiling of kitchens, dining rooms, storage rooms or other parts of the establishment should be constructed in the way that could prevent contamination of food due to dirty work environment, cross contamination of food utensils and infestation of vectors and rodents (21). Based on the result from this study cleanliness of wall and ceiling were found in a good conditions during inspection in 11 of kitchen and dining room respectively. Similarly all 12 premises' kitchen had openable window, adequate light and adequate ventilation which were in a better condition than a similar study conducted in Mekele, Awash Sebat-Kilo, and Zeway (1, 22, and 23). The repair condition and cleanliness of dining room were in a better condition than the kitchens. Perhaps this could be resulted due to majority of housekeepers spend their all-time through cleaning the dining room and give more consideration for it.

Each student catering establishment had to be provided with adequate, conveniently located toilet facilities accessible to the employees at all times to prevent occurrence of food borne outbreaks due to contamination of foods by employers or by insects (24). Thus all, 12, of establishments in AAU students catering had toilet facility. About 11 of toilets were clean during inspection.

All catering establishments need adequate washing facilities for staff. As well they must be provided with a sufficient number of suitable hand wash basins for the use of all persons engaged in the handling of food on or around the premises, and the basins should be conveniently placed in an accessible position. It also should be provided with an adequate supply of hot and cold water. Finally soap or other suitable detergents, and clean towels or other drying facilities must also be supplied (25). However, the findings of this study reveal that only 7 of student catering establishments had lavatory facility for food handlers. But, 6 , of those establishments with lavatory services had adequate supply of soaps and other detergents during inspection time.

Suitable provision must be made for the removal and 
storage of garbage and refuse originated in the premises. Waste storage place must be kept appropriately clean and a sufficient number of watertight containers of durable and with tight fitting or self-closing lids shall be provided for the storage of food waste and other refuse (25). According to the result half, 6 , of premises had container for different waste storage and all, 6 , of the establishments had a proper waste storage container. Although availability and condition of solid waste storage container in AAU much better than other private establishments it was very much low according to the reality. Because the remaining 6 of premises had not any temporary solid waste storage container during inspection time as a result this condition may result breading of insects and rodents and it could lead to cross contamination.

In this study area neatly all, 11, of inspected student catering establishments had adequate running water in kitchen house and all, 11, of them had both cold and running water at the same time. Since those student caterings were governmental and serve large number of students there should be more consideration to enhance continuous supply of water more than this.

If you clean food utensils manually a sink with at least three compartments shall be provided for washing, rinsing, and sanitizing equipment and utensils. Each compartment of the sink shall be supplied with hot and cold potable running water (25). In this study almost all 10 of establishments use machine to wash soiled dish and only 2 of them wash manually. Those 2 of establishments which wash utensils manually had a compartmentalized sink. However, none of catering establishments in other study areas use machine to wash food utensils rather they use manual hand washing system. Based on the findings, only half, 54.2\%, of catering establishments had three compartment sinks in Awash Sebat-Kilo in which is much lower. Majority, 11(91.7\%), of surveyed student caterings were use hot water and detergents for washing dishes which was better than a similar study conducted in Mekele in which only $8.1 \%$ of surveyed catering establishments use hot water to wash food utensil.

Foods should be protected from time and temperature abuse by purchasing according to the storage space available. Keep food in rooms designated for storage to prevent spoilage of food (26). Thus, in this study result 11 of premises has storage room for perishable as well for nonperishable foods. Similarly all 12 of establishments had walking type refrigerator during inspection time. In all, 12 $(100 \%)$, of the premises there was no any observed sign of spoilage.

Food handlers who had a better level of formal education had a good food handling and preparation practice than those who had lower level of formal education (27). Thus all of them had taken a formal education program whether higher or lower. This result reveals that it was in a better condition than a result obtained in Bahir dar, Mekele and India Solapur, in which $8.1 \%, 16.2 \%$ and $22.9 \%$ of them had no formal education respectively.
Knowledge and experience of food handlers regarding basics of food hygiene is a pillar to keep food safe and wholesome (28). Employees working in institutional establishments like in universities had the possibility of having satisfactory knowledge about food hygiene and personal hygiene. So, result of this study indicates that knowledge of food handlers regarding food borne disease was in a better condition but not about its cause and mode of transmission. Nearly all, 283(93.7\%), of food handlers in this study had adequate knowledge about food borne diseases. This study is similar to a study conducted in Mekele town and Indian college of medical science in which $89.5 \%$ and $95.2 \%$ were aware about food borne disease respectively. Having adequate knowledge regarding food borne disease transmission mechanisms and ways of food contamination is essential to undertake certain basic practice to alleviate those problems (28). In this study 245 $(81.1 \%)$ responded that contaminated food causes food borne disease and $214(70.9 \%)$ were responded that dirty hand was the main cause of food contamination. However, the practice of food handlers was not in a better condition unlike to their knowledge regarding food borne disease and modes of transmission. According to the result from their overall practice score nearly half of food handlers were practicing in poor conditions (Table-9). This perhaps resulted due to negligence of food handlers to follow the right practice in their work, the shortage of time to supply food for large number of students on time, inadequate supply of basic sanitary facilities, lack of food safety emphasis on food safety management and food safety education and training condition in the establishments (28, 29).

Food handlers should have to wear gown and aprons during food preparing and serving time to prevent food contamination and also dirty clothing could also be a source of microorganisms. Food workers who inadvertently touch their dirty clothing may contaminate their hands (29). But in this study the result showed that the number of food handlers who wear gown during work time was only 210 $(69.5 \%)$ indicating that lower than a similar study conducted in Mekele town which was $72.6 \%$ and also it is much lower than a study conducted in Bahir Dar (92.6\%) and Awasa (86\%) worn gown during inspection but it was in a better condition than a study conducted in Addis Ababa (54.2\%) of them had gown. However, half 160 (53\%) of them covered hair with restraints in which much better than similar studies conducted in Zeway (40.1\%), Mekele (39\%) and Awasa (11.8\%) but lower than the study conducted in Kenya (73\%) (30). Nearly half, 179(59.5\%), of them were without any finger ornaments during the time of inspection. The rate of food handlers who worn finger ornaments in Addis Ababa University were higher than that of the study conducted in Mekele town (41.5\% versus 35.7\%). Compared to other parts of the hand the area beneath fingernails harbor the most microorganisms and is the most difficult to clean (29). Majority, 219(72.5\%), of them were trimmed their fingernails short and almost all of them, 295 
(97.7\%) wash hands always before they start any activity which is not sufficiently enough.

Safer food preparation and handling were reported by person who took training on food preparation and handling and on those who had a better educational rank $(31,32)$. A study done in Costa Rica shows that lack of food safety training and certification and lack of knowledge and supervision were the prominent factors influencing the possibility of offering safe food. About, 197(65.2\%) of food handlers took training related with safe food handling and preparation practice which is much better than study conducted in Bahir Dar (21.8\%). Even if education, training, and the development of food safety certification examinations are key components in the process of ensuring that food handlers are proficient in and knowledgeable about food safety and sanitation principles the result indicated that amount of training provided for food handlers in this establishment were not enough. Besides, univariate and multivariate analysis result showed that, training status and educational status of food handlers had a clear influence on the practice score of food handlers. Therefore consideration should be given by university administrators to provide training periodically and checking their training status during recruitment.

Most of the time food handlers are a sensitive group of population who are the main focus of contamination since they are in direct contact with clients and food contact surfaces specially if they are in asymptomatic stage (33). A result from physical examination showed that only $1(0.3 \%)$ had cough during the time of inspection which were much lower than a study result obtained in Awash Sebat-Kilo in which visible skin infections were observed on two food handlers and ear infections were also found on another couple of food handlers, raising the infection rate to about $8.2 \%$ and also $9 \%$ of food handlers had health problem during inspection time in Mekele town. The positive actions in this university which need to be promoted were food handlers with any infectious disease could not be allowed to have any activity which had contact with food.

The presence of indicator organisms in a high counts in food contact surfaces, equipments and utensils have direct associations with cleaning efficiency and hygiene status of working environment $(34,35)$. The isolation of indicators like Staphylococcus aureus (S. Aureus), Escherichia coli (E. coli), coliforms and aerobic mesophilic bacteria (AMB) with a count of $>100$ colony $150 \mathrm{~cm}^{2}$ of swabbed utensils (above the acceptable level) gives a clear indication of feacal contamination with the risk of other pathogens that may be transmitted through water or food. According to the result from swab test the cleanliness of food utensils were in a better condition than a result obtained in Mekele town in which E.Coli and S.aureus were isolated from 18.2\% and $27.3 \%$ of the swabbed utensil respectively and it was comparable with the study conducted in south Ethiopia (36). This result perhaps indicates that the dish washing facility, water accessibility and other conditions which were available within the university students catering establishments were satisfactory as much as a required level. Acid and gas formation in 7(19.4\%) of the examined utensils would also confirm the presence of total and faecal coliform. However the isolation of Staphylococcus aureus gives the existence of poor hygiene of the food handlers who served as sources of contamination. This contamination was possibly due to human contact through air particles breathed, coughed or sneezed out during the course of work. As well as the presence of certain food handlers with certain infectious diseases could be the source of those identified S. aureus in swabbed food utensils.

\section{Strengths and Limitations of the Study}

\subsection{Strengths}

Laboratory analysis were made for detecting microbial quality of food utensils could increase the quality of data

\subsection{Limitations}

Facility based analysis was not performed due to small sample size of premises. Since data collection was done mostly by interviewing good practices of food handlers could be falsely inflated

\section{Conclusion}

Repair and sanitary status of premises were in a better condition in inspected establishments. There was poor accessibility and cleanliness of latrine service in some cafeterias. The overall hygiene practice of food handlers depicts that most of them had poor food handling practice. Food handlers with better educational status and training had better practice score. Due to presence of adequate dish washing facility the microbial quality of food utensils were in a better condition. Provision of training and basic sanitary facilities by management is recommended.

\section{References}

[1] Kinfe $Z$ and Abera K. Assessment of the sanitary condition of food and drink establishments in Mekele town. Ethiop.J.Health Dev. 2007;21(1): 3-11

[2] Jarmila S., Babak V., Martina H.,et al. Microbial contamination after sanitation of food contact surfaces in dairy and meat processing plants. Czech J. of food science. 2010;28(5): 450-461

[3] Laura R. G. and Carol S. Factors impacting food workers' and managers' safe food preparation practices: a qualitative study. J. of food protection trends. $2005 ; 25$ (12): 981-990

[4] Prianka M., Gautam J., Kanad B., et al. Identifying key risk behaviors regarding personal hygiene and food safety practices of food handlers working in eating establishments located within hospital campus of Kolkata, India. Al Ame en J Med S c i.20 1 2);5 (1 ) :2 1 -2 8 
[5] Alemayehu W/C and Mezgebu Y: Food hygiene lecture notes for environmental health part II. University of Gondar. May 2003

[6] Mulugeta K. and Bayeh A. The sanitary condition of food service establishments and food safety knowledge and practices of food handlers in Bahir dar town. Ethiop J Health Sci. 2012 ; 22(1): 27-35.

[7] Daniel N. and Abera K. Food hygiene practice and prevalence of intestinal parasites among food handlers working in Mekele university students caffeteria. J. of social science. 2012; 1(4): 065-071

[8] T/mariam S., Roma B., Sorsa S. and Erosie L. Assessment of sanitary and hygienic status of catering establishments of awasa town. Ethiop.J.Health Dev. 2000; 14(1): 91-98

[9] Fisseha G., Berhane Y. and GeberEmanuel T. Public catering establishments in Addis Ababa: physical and sanitary facilities. Ethiop.J.Health Dev. 1999; 13(2): 127-134

[10] Gashaw A., Afework K., Feleke M., Moges T. and Kahsay H. Prevalence of bacteria and intestinal parasites among food handlers in Gondar town, North West Ethiopia. J. of health, population and nutrition. 2008; 26(4): 451-455

[11] Paola P. and Allan O. Employees' food safety knowledge and practices in foodservice operations serving high risk populations. J. of the American Dietetic Association $2010 ; 120(10): 1-10$

[12] Shawndelle N., Mansel G., Sylvanus T. and Tanya M.: Frequency and type of food safety infractions in food establishments with and without certified food handlers in Canada. J of food protection trends. 2009; 29(12): 840-848

[13] Sara K.W. Food safety knowledge and practices of food recovery agency workers before and after food safety training. In Human ecology. Texas: Texas Christian university, 2004.

[14] Aisling M. and Declan B. Food safety knowledge, microbiology and refrigeration temperature in restaurant kitchens on the island of the Ireland. Journal of the national food centre of Ireland. Journal of Food Protection. 2010; 68 (7): $1421-1430$.

[15] Anant A. and Anjali.P.K. Assessment of personal hygiene of canteen workers of government medical college and hospital in solapur, India. National J. of community medicine. 2011; 2(3): $448-451$

[16] Laura R.G., Vincent R., Ryan M., et al. Factors related to food workers hand hygiene practices. J. of food protection trends. 2007; 70(3): 661-666

[17] Brita B., Anne W. and May A. Background factors affecting the implementation of food safety management systems. Canada J of food protection. 2013; 30(2): 078-086

[18] Barrash J. An assessment of food safety knowledge and practices of catering employees in Nevada Las vegas, USA. British food journal. 2007; 109(7): 562-576

[19] Begani R., Tombe B., Polong T. Effectiveness of cleaning and sanitation of food contact surfaces in the PNG fish canning industry. DWU research journal. 2012; 16(3)

[20] Kiiyukia, D.C. Laboratory manual of food microbiology for Ethiopian health and nutrition research institute. Ethiopia, Addis Ababa, Dec, 2003.
[21] Sanitary standards for food establishments: Current with material published in Conn.L.J. through 6/10/08 (Effective June 22, 1990- September 1, 2005.)

[22] Abera K., Genete H., Kebede E. et la. The sanitary condition of public food and drink establishments in the district town of Zeway. Ethiop.J.Health Dev. 2002; 16(1): 95-104

[23] Abera K., Ashebir M., Aderajew A., et al. The sanitary condition of food and drink establishments in Awash SebatKilo, afar region. Ethiopia. Ethiop.J.Health Dev. 2006; 20(3): 201-203

[24] Colorado retail food establishment rules and regulations, adopted by the state board. 2006. http://www.cdphe.state.co.us/op/regs/consumer/101019.pdf,)

[25] Food hygiene in catering establishment: legislation and model regulations. Geneva, Switzerland WHO in collaboration with UNEP, 1977

[26] Durham university food safety manual,reviewed : January $2006 / \mathrm{http} / /$ www.dur.ac.uk/resource/healthandsafety/manual/

[27] Impact study: Essential skills and food sanitation and hygiene training. December 2004. http://www.nald.ca/AWES/pdf

[28] Grassi M.A., Osella E, Civera T., et al. Analysis of food safety and hygiene in mass catering establishments:. Journal of Industie Alimetari.2008. 47(480), 471-478

[29] Angela M. Safe food handlers manual, June 2009. http://www. angela fraser@ncusu.edu

[30] Faith Muhonja, George Kobia Kimathi. Assessment of Hygienic and Food Handling Practices among Street Food Vendors in Nakuru Town in Kenya. Science Journal of Public Health. Vol. 2, No. 6, 2014, pp. 554-559. doi: $10.11648 / j . s j p h .20140206 .19$

[31] Mohamed F., Naglaa F., Wafaa M. and Bakr K., assessment of hand washing facilities, personal hygiene and the bacteriological quality of hand washes in some grocery and dairy shops in Alexandria, Egypt.. Journal of Alexandria University. 2009; 84 (1 \& 2)

[32] Angelillo, I.F., Viggiani, N.M.., Rizzo L. \& Bianco A.: Food handlers and foodborne diseases: knowledge, attitudes, and reported behavior in Italy. Journal of Food Protection. 2011; 63. 381-385.

[33] P. Legnani a, E. Leonia, M. Berveglieri ,et al. Hygienic control of mass catering establishments, microbiological monitoring of food and equipment, in Bologna , German .:Journal of food Control. 2011; 15 (24). 205-211

[34] International standard organization ISO 18593. 2004(E). Horizontal methods for sampling techniques from surfaces using contact plates and swabs

[35] International standard organization ISO 4832, 2006(E). Microbiology of animal feeding stuffs-horizontal method for the enumeration of coliform colony count techniques

[36] Misganaw Birhaneselassie, David Williams. A Study of Salmonella Carriage among Asymptomatic Food-Handlers in Southern Ethiopia. International Journal of Nutrition and Food Sciences. Vol. 2, No. 5, 2013, pp. 243-245. doi: $10.11648 / j . i j n f s .20130205 .15$ 